\title{
Les lubrifiants secs pour le tréfilage
}

\section{Céline GUERIN}

CONDAT Lubrifiants,

104 avenue Frédéric Mistral,

38670 Chasse sur Rhône,

France

<celine.guerin@condat.fr>

\begin{abstract}
The wire drawing process consists in reducing the diameter of a metallic wire by traction through a die. Wire drawing dry lubricant is a complex product not only considering the selection of raw materials, theirs formulation but also the manufacturing process. It can be assimilated to an elaborate mixture of mainly sodium and calcium soaps with mineral elements (fillers) and additives. The composition and the association of these elements allow the control of thickness and consistence of the lubricant film through the dies. Fatty matters mainly used for the manufacturing of these soaps are tallow and stearic acid extracted from tallow. Initially considered as a waste and questioned during the BSE crisis, tallow is nowadays on demand especially on the biofuels market.
\end{abstract}

Key words: Soaps, wire drawing, lubricant, pre-coatings

contact, mais également des traitements de surface qui vont favoriser l'accroche du savon sur les fils métalliques. Ces savons commercialisés à l'international sont fabriqués sur trois sites industriels : au siège social de Chasse-sur-Rhône (Nord-Isère) et dans les deux filiales implantées aux États-Unis et au Brésil.

\section{Données économiques}

Le marché du suif a largement évolué depuis les dernières décennies. Initialement considéré comme un sous-produit, de nombreux travaux ont été entrepris pour le valoriser et il est passé rapidement au rang de matière première industrielle.

L'histoire de Condat a débuté en avec un jeune Auvergnat qui est venu s'installer à Gerland, dans les faubourgs de Lyon, pour faire le commerce de suifs et autres résidus de boucheries. À la révolution industrielle, la demande de produits pour graisser les machines augmente, notamment pour les locomotives de la compagnie de chemin de fer Paris-Lyon-Marseille dite "PLM ». À cette époque, on ne connaissait pas les huiles et graisses minérales à base de pétrole, mais seulement le suif, pur ou mélangé à de l'huile végétale de colza en particulier. La diversification de la production pour répondre à la demande des industriels est devenue une priorité des héritiers du fondateur. Ainsi Condat développe la fabrication de savons, de graisses calciques, de sulforicinates et de briquettes de laminoirs pour commencer. Dans les années 1950, Condat commence à travailler sur la fabrication de savons secs pour le tréfilage de fils métalliques. Après de constantes recherches et améliorations, Condat est aujourd'hui le leader mondial sur ce métier en proposant les savons secs qui vont lubrifier les pièces métalliques en

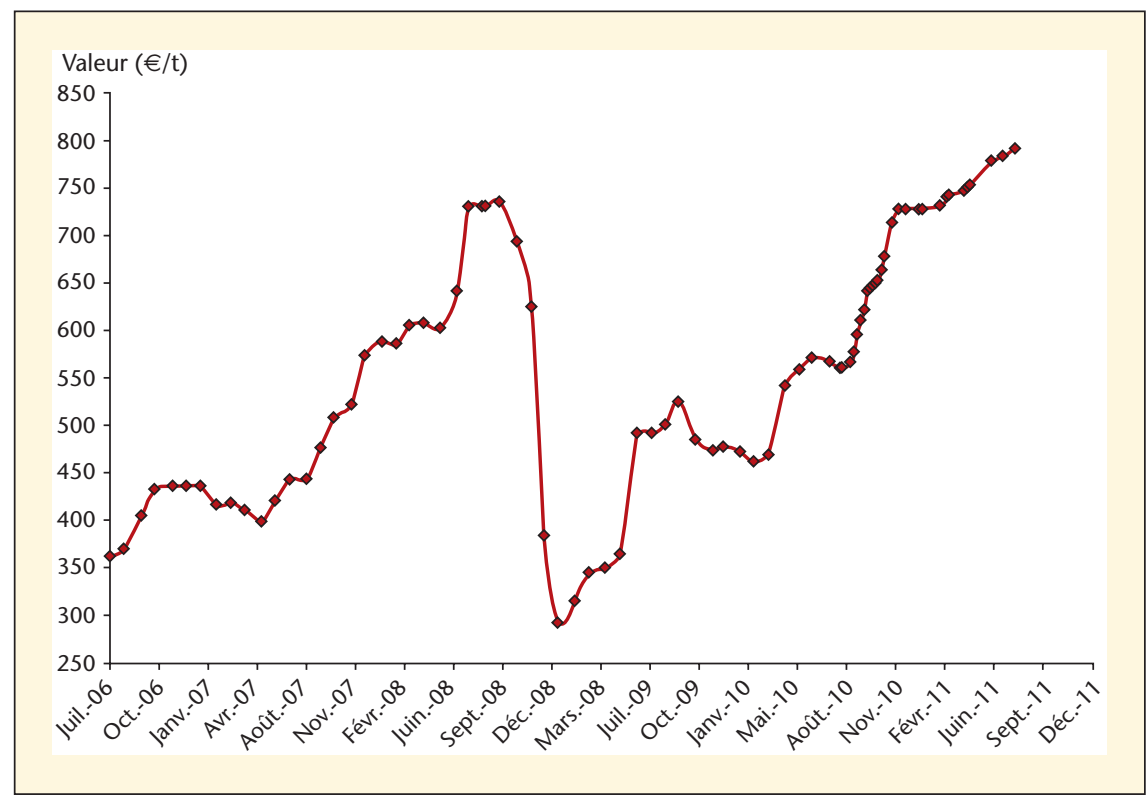

Figure 1. Évolution du cours du suif à la bourse de Milan sur les cinq dernières années.

Pour citer cet article : Guerin C. Les lubrifiants secs pour le tréfilage. OCL 2012 ; 19(1) : 51-53. doi : 10.1684/ocl.2012.0429 


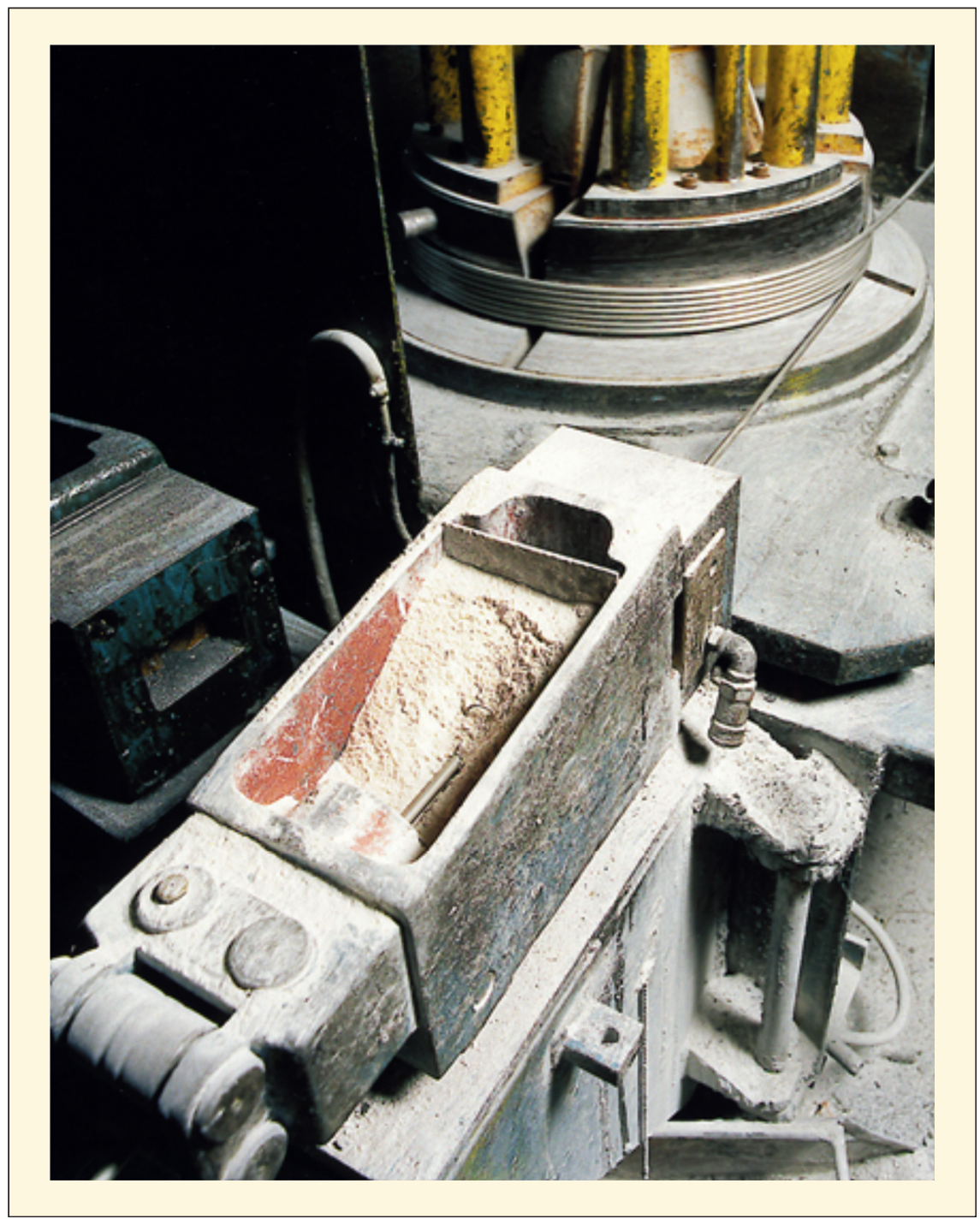

Figure 2. Boîte à savon.

Puis, les suspicions de transmission de l'encéphalite spongiforme bovine (ESB) à I'homme ont fait chuter son cours dans les années 2000. Depuis, une systématisation des tests de dépistage de I'ESB à l'abattage et une meilleure connaissance de l'obtention de ces produits ont permis de réhabiliter le suif auprès de l'opinion publique, au moins pour l'industrie nonalimentaire. En effet, il ne faut pas confondre " suif » et " graisses animales ", le suif issu du gras de bœuf est produit dans des ateliers particuliers appelés fondoirs, à partir de la fonte de tissus adipeux des animaux qui sont considérés comme des "gras nobles".

Le marché du suif se partage à I'heure actuelle entre 3 types $d^{\prime}$ industries :

reusement, ceci a nettement changé donne en termes de demande et a eu pour effet de doper le prix du suif (figure 1). Certains pays commencent à remettre en cause ces réformes qui incitent de plus en plus les industriels à se tourner vers la stéarine de palme. Cependant, la stéarine de palme n'a pas permis de réduire le cours du suif du fait d'une demande toujours croissante en biodiesel. Elle a juste permis d'accroître la disponibilité mondiale en stéarine.

\section{Évolutions règlementaires}

Condat est pro-actif en termes de respect des dernières règlementations du secteur de la chimie sur la santé et la sécurité (Directive Biocides, Règlement REACH, nouveaux classements des substances, etc.) grâce à son département "Affaires réglementaires ". L'équipe est fortement impliquée et participe à de nombreux consortiums européens, maximisant ainsi l'anticipation sur les développements $R \& D$ à venir.

La philosophie de Condat en matière de $R \& D$ est la recherche systématique de substituts aux matières premières potentiellement dangereuses SVHC (substance of very high concern). À titre d'exemple, la réglementation sur le borax $^{1}$ a conduit l'équipe de R\&D dédiée au tréfilage à reformuler ses lubrifiants pour respecter et même anticiper toute future évolution tout en préservant leur efficacité dans les procédés des clients. Elle propose à présent une plus large gamme de produits de tréfilage pour une substitution immédiate : traitements de surface et savons sans borax ou à faibles taux de borax.

Dans le cadre de sa contribution au développement durable, et d'une démarche d'éco-conception, Condat propose une large gamme de produits de très hautes performances (graisses de maintenance, huiles entières ou solubles pour l'usinage des métaux) formulés sur bases naturelles. Certains produits certifiés Ecolabel ont été conçus pour répondre à la loi du 5 janvier 2006 visant à encourager le développement de produits biodégradables pour I'utilisation dans des zones naturelles sensibles. II s'agit d'un fluide hydraulique "Bio Natur Hydrolabel », d'une huile pour moteur 2 temps "Bio Natur 2 TPS " et de 2 huiles pour chaînes de tronçonneuses individuelles et d'abatteuses mécaniques "Bio Natur Chain 100 " et "Bio Natur Chain Pro ».

\footnotetext{
${ }^{1}$ Tétraborate de sodium décahydraté de formule $\mathrm{Na}_{2} \mathrm{~B}_{4} \mathrm{O}_{7} .10 \mathrm{H}_{2} \mathrm{O}$.
} 


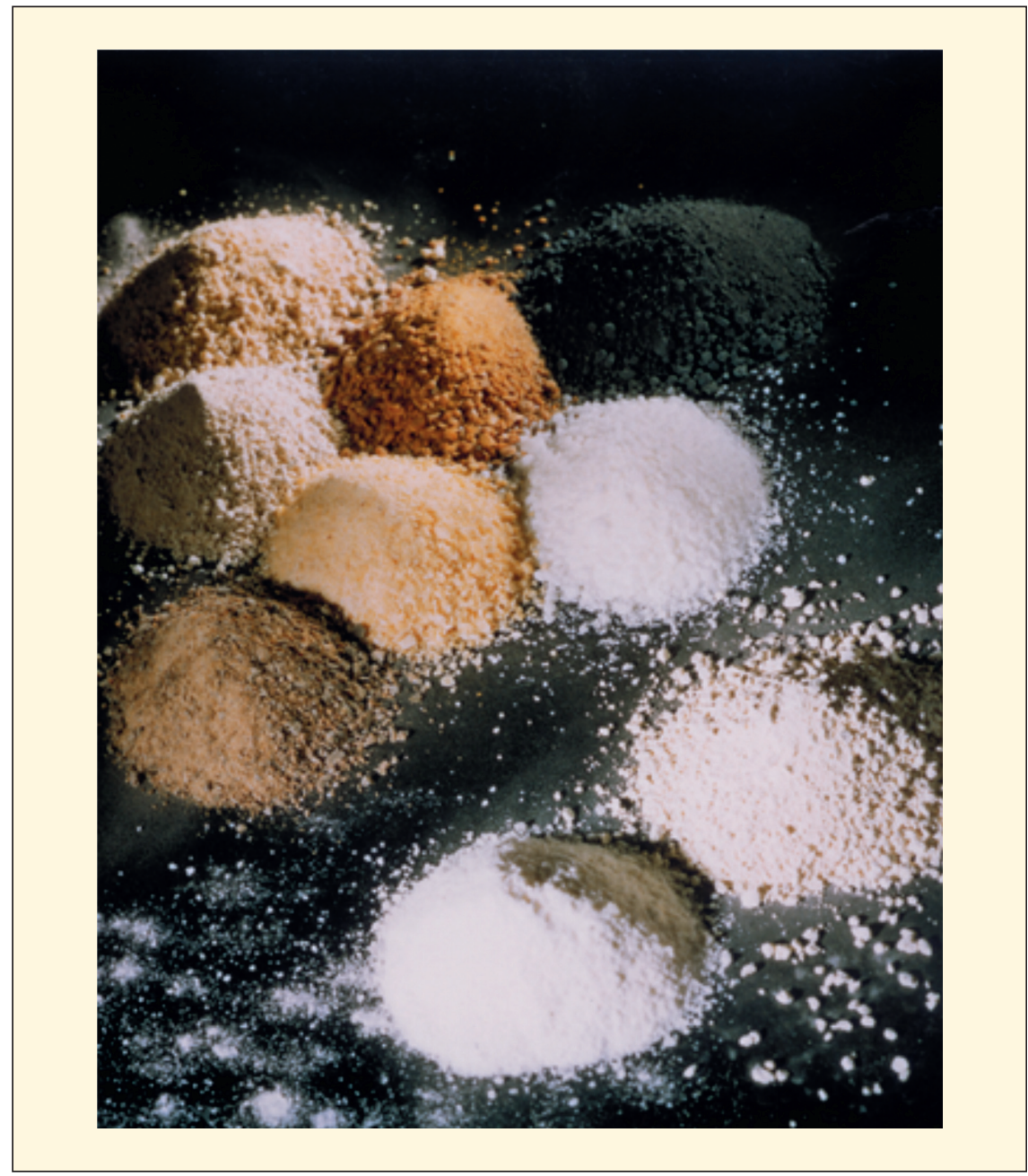

Figure 3. Savons de tréfilage de chimies et de granulométries différentes.

\section{Lien entre les matières premières et les propriétés physico- chimiques des savons de tréfilage}

Il faut considérer globalement les lubrifiants secs pour le tréfilage comme des mélanges complexes de savons de calcium ou de sodium avec des composés minéraux permettant de réguler l'épaisseur et la consistance du film, et des additifs. En effet, le lubrifiant sec doit offrir un film épais pour pouvoir couvrir la rugosité du fil et obtenir une bonne séparation entre le fil et la filière.

Les matières grasses utilisées pour la fabrication des savons de tréfilage sont principalement le suif et I'acide gras de suif donnant une répartition majoritaire en acides gras insaturés (35-40\% de bonne reproductibilité des lubrifiants formulés.

La matière grasse n'est pas le seul critère clé pour formuler un savon de tréfilage répondant aux conditions d'utilisation. En effet, les deux grandes familles de savons (calciques ou sodiques) ont des comportements très différents du fait de leur structure bivalente pour les calciques et monovalente pour les sodiques. Un savon de sodium à teneur en matière grasse identique donne un film lubrifiant plus mince qu'un savon de calcium qui, lui, est moins résistant à la température et au cisaillement. À titre d'exemple, on utilisera plutôt des savons de calcium à faible teneur en matière grasse pour le tréfilage d'acier doux décalaminé nécessitant une charge importante du fil, alors que des savons de sodium à haute teneur en matière grasse seront préférés pour le tréfilage d'acier dur avec fini brillant. Les stéarates de sodium du fait de leur structure chimique sont plus performants mais exigent une bonne préparation de surface.

La granulométrie d'un savon de tréfilage est aussi un paramètre essentiel. En effet le mouvement de la poudre dans la boîte à savon (figure 2) est directement conditionné par ses caractéristiques physiques de forme et de taille de grains (figure 3). Une poudre trop fine peut favoriser la formation de tunnels alors qu'une poudre trop grosse est difficilement entraînée dans le cône d'entrée de la filière. II faut éviter la présence de grumeaux en amont de la filière limitant ou stoppant I'alimentation en lubrifiant. Dans la boîte à savon, le lubrifiant circule, d'où une modification de la granulométrie par une action de broyage. La forme initiale des particules de savon est essentiellement donnée par le procédé de fabrication.

\section{Conclusion}

température à laquelle le lubrifiant passe de l'état solide à l'état visqueux. Ce comportement est influencé par la présence de glycérol, les types de savons formés et surtout par le procédé de production. Le paramètre le plus difficile à verrouiller est l'approvisionnement des matières grasses utilisées par fabriquer les savons. En effet, ce sont des matières premières de qualité technique qui évoluent en fonction des fournisseurs et des lots. Il est donc nécessaire de resserrer au maximum les critères d'acceptation de ces matières grasses pour obtenir une
Les savons secs de tréfilage sont donc des produits très techniques du fait des différents paramètres imposés par les conditions de tréfilage. Il est nécessaire de connaître parfaitement les matières premières et de maîtriser le procédé de fabrication pour produire des savons convenant parfaitement aux conditions de tréfilage et aux évolutions réglementaires visant à réduire voire peut-être à terme interdire totalement I'utilisation de borax. 